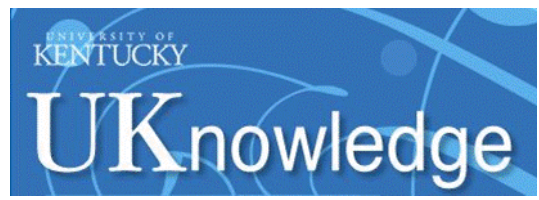

University of Kentucky

UKnowledge

2-23-2015

\title{
Temperature Dependence of Anisotropic Magnetoresistance in Antiferromagnetic $\mathrm{Sr}_{2} \mathrm{IrO}_{4}$
}

C. Wang

University of Texas at Austin

H. Seinige

University of Texas at Austin

Gang Cao

University of Kentucky, cao@pa.uky.edu

J.-S. Zhou

University of Texas at Austin

J. B. Goodenough

University of Texas at Austin

See next page for additional authors

Follow this and additional works at: https://uknowledge.uky.edu/cam_facpub

Part of the Materials Science and Engineering Commons, and the Physics Commons

Right click to open a feedback form in a new tab to let us know how this document benefits you.

\section{Repository Citation}

Wang, C.; Seinige, H.; Cao, Gang; Zhou, J.-S.; Goodenough, J. B.; and Tsoi, M., "Temperature Dependence of Anisotropic Magnetoresistance in Antiferromagnetic $\mathrm{Sr}_{2} \mathrm{IrO}_{4}$ " (2015). Center for Advanced Materials Faculty Publications. 2.

https://uknowledge.uky.edu/cam_facpub/2

This Article is brought to you for free and open access by the Center for Advanced Materials at UKnowledge. It has been accepted for inclusion in Center for Advanced Materials Faculty Publications by an authorized administrator of UKnowledge. For more information, please contact UKnowledge@lsv.uky.edu. 


\section{Temperature Dependence of Anisotropic Magnetoresistance in Antiferromagnetic $\mathrm{Sr}_{2} \mathrm{IrO}_{4}$}

\section{Digital Object Identifier (DOI)}

http://dx.doi.org/10.1063/1.4913300

\section{Notes/Citation Information}

Published in Journal of Applied Physics, v. 117, no. 17, article 17A310, p. 1-4.

Copyright 2015 American Institute of Physics. This article may be downloaded for personal use only. Any other use requires prior permission of the author and the American Institute of Physics.

The following article appeared in Journal of Applied Physics, v. 117, no. 17, article 17A310, p. 1-4 and may be found at http://dx.doi.org/10.1063/1.4913300.

\section{Authors}

C. Wang, H. Seinige, Gang Cao, J.-S. Zhou, J. B. Goodenough, and M. Tsoi 


\title{
Temperature dependence of anisotropic magnetoresistance in antiferromagnetic $\mathrm{Sr}_{2} \mathrm{IrO}_{4}$
}

\author{
C. Wang, ${ }^{1,2}$ H. Seinige,${ }^{1,2}$ G. Cao,${ }^{3}$ J.-S. Zhou, ${ }^{2}$ J. B. Goodenough, ${ }^{2}$ and M. Tsoi ${ }^{1,2, a)}$ \\ ${ }^{1}$ Physics Department, University of Texas at Austin, Austin, Texas 78712, USA \\ ${ }^{2}$ Texas Materials Institute, University of Texas at Austin, Austin, Texas 78712, USA \\ ${ }^{3}$ Center for Advanced Materials, University of Kentucky, Lexington, Kentucky 40506, USA
}

(Presented 5 November 2014; received 22 September 2014; accepted 27 October 2014; published online 23 February 2015)

Temperature-dependent magnetotransport properties of the antiferromagnetic semiconductor $\mathrm{Sr}_{2} \mathrm{IrO}_{4}$ are investigated with point-contact devices. The point-contact technique allows to probe very small volumes and, therefore, to look for electronic transport on a microscopic scale. Pointcontact measurements with single crystals of $\mathrm{Sr}_{2} \mathrm{IrO}_{4}$ were intended to see whether the additional local resistance associated with a small contact area between a sharpened $\mathrm{Cu}$ tip and the antiferromagnet shows magnetoresistance (MR) such as that seen in bulk crystals. Point-contact measurements at liquid nitrogen temperature revealed large MRs (up to 28\%) for modest magnetic fields $(250 \mathrm{mT})$ applied within an $\mathrm{IrO}_{2}(a b)$ plane with angular dependence showing a crossover from four-fold to two-fold symmetry with an increasing magnetic field. Point contact measurement exhibits distinctive anisotropic magnetoresistance (AMR) in comparison to a bulk experiment, imposing intriguing questions about the mechanism of AMR in this material. Temperature-dependent MR measurements show that the MR falls to zero at the Neel temperature, but the temperature dependence of the MR ratio differs qualitatively from that of the resistivity. This AMR study helps to unveil the entanglement between electronic transport and magnetism in $\mathrm{Sr}_{2} \mathrm{IrO}_{4}$ while the observed magnetoresistive phenomena can be potentially used to sense the antiferromagnetic order parameter in spintronic applications. (C) 2015 AIP Publishing LLC. [http://dx.doi.org/10.1063/1.4913300]

The emerging developments of spintronics aim to tackle critical challenges in nowadays data storage technologies. So far, most magneto-electronic devices have been exploring the magnetism in ferromagnets (FMs) while antiferromagnets (AFMs) are mainly used in secondary roles such as exchange biasing FMs. ${ }^{1}$ However, AFM materials have some unique advantages over FMs such as a minimized cross-talk between AFM nanocrystals due to the absence of stray fields; possibly stronger spin-transfer torques, which is important for a better control of magnetic states in spintronics applications. ${ }^{2-5} \mathrm{~A}$ critical milestone towards the implementation of AFMs in spintronic devices is to find reliable ways to sense the local antiferromagnetic order. Anisotropic magnetoresistance $(\mathrm{AMR})^{6}$ and tunneling $\mathrm{AMR}^{7,8}$ observed in AFMs are promising candidates for this purpose. The iridate $\mathrm{Sr}_{2} \mathrm{IrO}_{4}$ (SIO), which has been found to have $J_{\text {eff }}=1 / 2$ magnetic moments ordered in a canted antiferromagnetic manner below its Neel temperature $(240 \mathrm{~K})$ is a particularly interesting material for such AMR studies because of a strong spin-orbit coupling and intriguing responses under external electric/magnetic fields. ${ }^{9,10}$ Recently magneto-electronic transport studies have reported magneto-electric phenomena in SIO crystals ${ }^{10,11}$ and thin films, ${ }^{6}$ suggesting that this $5 \mathrm{~d}$ transition metal oxide could provide a rich playground for studying the correlations between AFM magnetic-order properties, electron transport, and orbital physics.

\footnotetext{
${ }^{a}$ Author to whom correspondence should be addressed. Electronic mail: tsoi@physics.utexas.edu.
}

Here we report a point-contact (PC) study of temperaturedependent magnetoresistive (MR) phenomena in $\mathrm{Sr}_{2} \mathrm{IrO}_{4}$ (SIO) single crystals. Point contacts enable us to probe locally electron transport on a microscopic scale, and we intended to examine whether the local resistive probe in the small contact area between a sharpened $\mathrm{Cu}$ tip and the SIO crystal can sense MR signals similar to those seen in bulk measurements. The measurements at liquid nitrogen temperature revealed large MRs (up to 28\%) for modest magnetic fields ( $250 \mathrm{mT}$ ) applied within the $\mathrm{IrO}_{2}$ a-b plane. The MR ratios were found to drop to zero with increasing temperature up to $\mathrm{T}_{\text {Neel }} \sim 240 \mathrm{~K}$, confirming that the observed MR is associated with the response of magnetic order under external magnetic fields. Moreover, the angular dependence of MR exhibited an AMR behavior with an intriguing transition from 4-fold to 2-fold symmetry in response to an increasing magnetic field, which is tentatively attributed to a magnetic-fieldinduced change of the canted magnetic order in the $\mathrm{IrO}_{2}$ planes of the SIO Ruddlesden-Popper phase. ${ }^{12}$ Notably, AMR of point contacts showed a distinctive magnetic field dependence that is different from the AMR of the bulk crystal. Comparison of the point-contact AMR and the bulk AMR may help to unveil the domain dynamics under applied magnetic fields. Our observations also support potential applications of AMR phenomenon in AFM spintronics where it can be used to sense the magnetic order of AFMs.

The investigated single crystal of layered $\mathrm{Sr}_{2} \mathrm{IrO}_{4}$ $(1.5 \mathrm{~mm} \times 1 \mathrm{~mm} \times 0.5 \mathrm{~mm})$ was synthesized via a self-flux technique. ${ }^{13} \mathrm{Sr}_{2} \mathrm{IrO}_{4}$ crystallizes in a reduced tetragonal 
structure with a unit cell of 4 layers of $\mathrm{IrO}_{6}$ as a result of a rotation of the $\mathrm{IrO}_{6}$ octahedra about the c-axis by $\sim 12^{\circ}{ }^{14} \mathrm{It}$ has been known that $J_{\text {eff }}=1 / 2$ moments of the $5 \mathrm{~d}$ electrons of $\mathrm{Ir}^{4+}$ tend to lie in the $\boldsymbol{a b}$-plane and approximately follow the rotation of the $\mathrm{IrO}_{6}$ octahedra, thus forming a canted antiferromagnetic order below the Neel temperature of about $240 \mathrm{~K}$. The SIO magnetic order can experience a meta-magnetic transition where weakly ferromagnetic residual moments of $\mathrm{IrO}_{6}$ planes can be saturated by an externally applied magnetic field above the critical field $B_{c} \approx 200 \mathrm{mT} .{ }^{15}$ We performed magneto-electronic transport measurements on the microscopic scale of SIO by utilizing PC devices. Mechanically controlled point contacts ${ }^{16}$ were made between sharpened $\mathrm{Cu}$ tips and the (001) surface of a SIO single crystal; this experimental geometry enables us to inject electrical currents locally into the crystal with current flowing (primarily) along the 001 $c$-axis into a macroscopic $\mathrm{Cu}$ electrode on the back of the sample (see inset to Fig. 1). The PC system used in this study can produce contacts with cross-sectional dimensions $a$ from microns down to a few nanometers, which can be estimated from the diffusive model of electronic transport in such PCs using the PC resistance $R=\rho / 2 a{ }^{17}$ Given the resistivity $\rho$ $\approx 50 \Omega \mathrm{cm}$ of $\mathrm{SIO}$ at $\mathrm{T}=77 \mathrm{~K}$, the observed resistance ranging from $R=15 \mathrm{k} \Omega-1.4 \mathrm{M} \Omega$ yields $a \sim 45 \mathrm{~nm}-4.2 \mu \mathrm{m}$. MR of such PCs was measured with magnetic fields (up to $250 \mathrm{mT}$ ) applied in the basal ( $\boldsymbol{a} \boldsymbol{b}-$ ) plane of the perovskite. As is shown in Fig. 1, negative MRs (i.e., resistance decreases in the presence of magnetic field) with hysteresis were observed at $\mathrm{T}=77 \mathrm{~K}$ (top trace in Fig. 1). The hysteresis in MR is supposedly following the hysteresis in sample magnetization coming from the saturated residual moments of IrO planes. MRs were found in all of contacts investigated with highest MR ratios $\left(R_{\max }-R_{\min }\right) / R_{\min }$ up to $28 \% .{ }^{12}$ Here we focus on the temperature dependence of PC resistance and MR measured at temperatures $\mathrm{T}$ from $77 \mathrm{~K}$ to $295 \mathrm{~K}$, covering the Neel temperature of magnetic phase transition at $240 \mathrm{~K}$. We also compare the PC behavior with bulk transport measurements (see

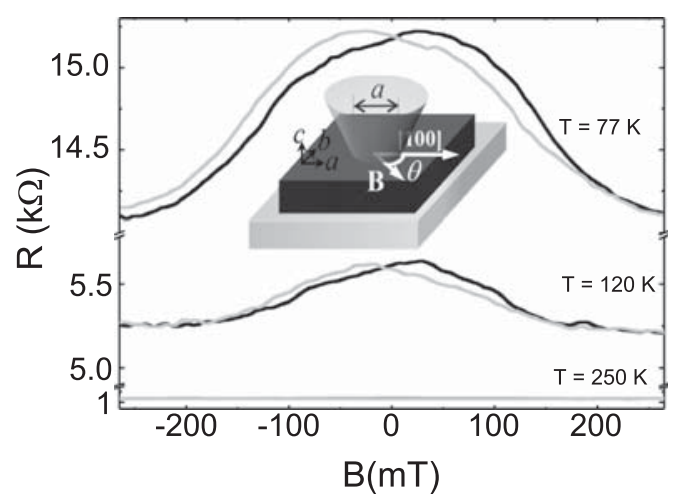

FIG. 1. Magnetoresistance of SIO measured by point-contacts at 3 different temperatures $(\mathrm{T}=77 \mathrm{~K}, 120 \mathrm{~K}$, and $250 \mathrm{~K})$. One can see that the MR signal has vanished at $\mathrm{T}=250 \mathrm{~K}$ (above $\mathrm{T}_{\mathrm{Neel}} \sim 240 \mathrm{~K}$ ). Black (grey) curves correspond to up (down) sweeps of applied magnetic field. The vertical-axis scale is the same for all 3 curves. Inset shows the experimental geometry: sharpened $\mathrm{Cu}$ tip (top grey cone) is in contact with SIO single crystal (center black block) backed by a $\mathrm{Cu}$ plate (bottom grey). The applied magnetic field $\boldsymbol{B}$ is rotated in the $\boldsymbol{a} \boldsymbol{b}$-plane thus changing the angle $\boldsymbol{\theta}$ between $\boldsymbol{B}$ and the (100) axis.
Fig. 2(b)) performed on the same single crystal, which showed similar temperature dependence to that measured with PC (see Fig. 2(a)). For the bulk measurements the PC was replaced by a macroscopic silver-paste electrode with a crosssectional area $\sim 1 \mathrm{~mm}^{2}$.

The temperature dependence of PC (and bulk) resistance shows an exponential decay with increasing temperature that clearly reflects the semiconducting nature of transport in SIO. Note that our PCs to SIO are ohmic in nature ${ }^{12}$ as PC current-voltage (I-V) characteristics are linear at small biases applied; we have not observed any significant decreases in PC resistance vs bias which would be expected in the tunneling regime of transport between a metallic $(\mathrm{Cu})$ tip and a semiconductor with a relatively small gap (30-200 meV). From the $R$ vs $T$ measurement, one can obtain band-gap values $\Delta \sim 60 \mathrm{meV}$ following the model of thermally excited conductivity in semiconductors. The MR ratio decreases with increasing temperature until it vanishes at $\mathrm{T} \sim 240 \mathrm{~K}$, implying that MR should be associated with changes in magnetic order under externally applied fields. At the same time, as is shown in the inset to Fig. 2, the MR ratio decreases to zero approximately linearly, showing qualitatively different behavior as a function of temperature if compared to the exponential decay of $R$ vs $T$. It is known from previously reported field cooling (FC) data that saturated magnetic moments would not vary significantly at temperatures below $200 \mathrm{~K}^{10}{ }^{10}$ Therefore, the linear temperature dependence of MR ratio cannot be simply interpreted as an indication of a temperature effect on magnetic moments. According to the model that MR is the result of lattice distortions induced by the reoriented magnetic moments under fields, a rising MR ratios at low temperatures (well below $\mathrm{T}_{\mathrm{Neel}}$ ) implies that the influence of magnetism on lattice dynamics and electron transport may be stronger at lower temperatures.

The observed MR signals are found to be anisotropic when the direction of the applied magnetic field is varied within the $\boldsymbol{a} \boldsymbol{b}$-plane. The normalized AMR defined as $\left[R(\theta)-\mathrm{R}_{\min }\right] /\left[\mathrm{R}_{\max }-\mathrm{R}_{\min }\right]$, with $\theta$ being the angle between the applied magnetic field and the 100 a-axis (see the inset to Fig. 1), is shown in Fig. 3 with 2D density plots as a function of magnetic field strength $B$ and angle $\theta$. Note that the relative direction between the electrical current (injected along the c-axis) and the applied magnetic field remains essentially unchanged when the field is rotated in the $\boldsymbol{a} \boldsymbol{b}$-plane.
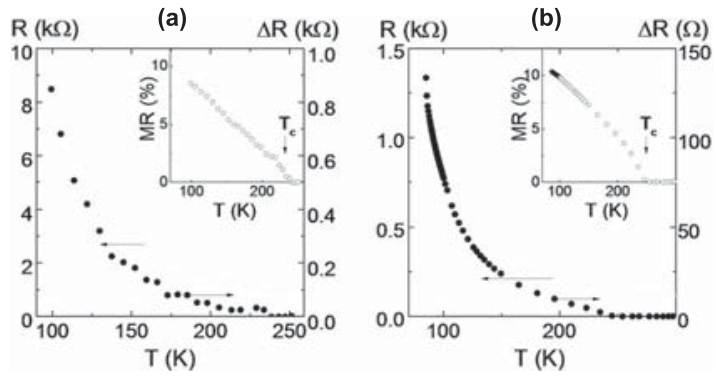

FIG. 2. Resistance $R$ (circles) and change of resistance $\Delta R$ (solid) vs temperature measured in (a) point-contact geometry and (b) bulk sample. The insets show the magnetoresistance $(\mathrm{MR}=\Delta R / R)$ ratio vs temperature in respective measurements. 
(a)

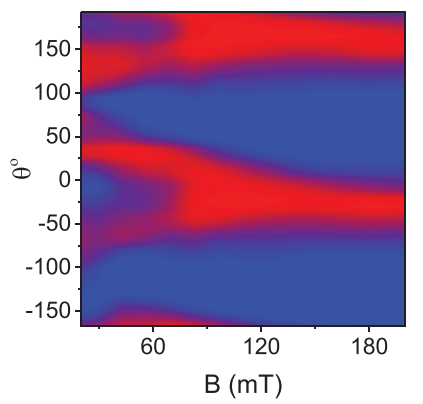

(b)

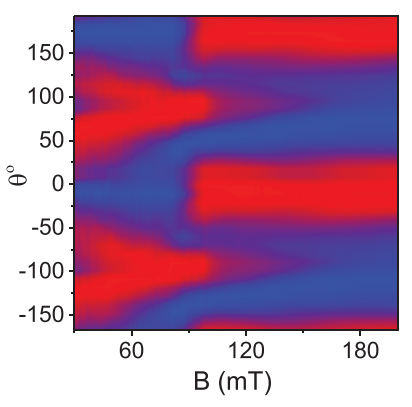

FIG. 3. 2D density plots (blue $=0$; red $=1$ ) of normalized AMR vs applied magnetic field $B$ and angle $\theta$ from (a) point-contact and (b) bulk measurements. All measurements were done at $\mathrm{T}=77 \mathrm{~K}$.

Therefore, we would like to point out that the AMR observed in our experiments cannot be explained by the conventional AMR in polycrystalline magnetic conductors, which is defined solely by the relative angle between the current direction and magnetic moments. Instead, it is the relative orientation between the crystal lattice and the applied magnetic field that is being altered when rotating magnetic fields in the $\boldsymbol{a} \boldsymbol{b}$-plane, and thus the observed AMR is attributed to a crystalline component of AMR. ${ }^{6}$ Similar AMRs have been found in all PCs studied. We found the AMR measured by point contacts can be as large as $14 \%$, which is very large compared to previously reported weak signals of crystalline AMRs in $3 \mathrm{~d}$ transition metal alloys/oxides $(0.1 \%-0.5 \%)^{18-20}$ and is also larger than crystalline AMRs observed to date in $\mathrm{Sr}_{2} \mathrm{IrO}_{4}{ }^{6}$

The angular dependence of MR $(\mathrm{T}=77 \mathrm{~K})$ exhibits an intriguing dependence on the strength of the applied magnetic field: the AMR starts with a 4-fold rotational symmetry at small fields ( $\sim 40 \mathrm{mT})$, and changes from 4 -fold to 2 -fold symmetry as the applied magnetic field ramps up towards saturation. As we show in Fig. 3(a), the symmetry of the AMR becomes mostly 2-fold at a relatively low field $(\sim 60 \mathrm{mT})$, indicating the predominance of uniaxial anisotropy upon the saturation of the uncompensated residual moments of $\mathrm{IrO}_{6}$ planes. Bulk AMR was also measured and analyzed as a function of $B$ and $\theta$ (to be compared with the PC AMR). It is found that bulk AMR is smaller and exhibits a qualitatively different field dependence as shown in Fig. 3(b). Although the field-induced changes in AMR symmetry are clearly present, there is a "boundary field" around $100 \mathrm{mT}$ that defines two distinct regimes of the observed AMR. In the low field $(B \lesssim 100 \mathrm{mT})$ regime, the field dependence of the AMR symmetry of the bulk qualitatively resembles that in PCs. On the other hand, in the large field $(B \gtrsim 100 \mathrm{mT})$ regime, where the PC AMR readily exhibits a 2-fold symmetry, bulk AMR shows a reminiscence of the 4fold symmetry up to $B \sim 150 \mathrm{mT}$ in the bulk. Another clear distinction is that the axis of maximum AMR experiences a step-like shift and becomes independent of the magnetic field magnitude up to the largest fields applied.

The observed AMR reveals the entanglement of magnetoelastic effects, spin-orbit coupling, and electron conduction. From previous studies, it is known that the magnetic

moments in $\mathrm{Sr}_{2} \mathrm{IrO}_{4}$ follow approximately the rotations of the $\mathrm{IrO}_{6}$ octahedra. ${ }^{22-24}$ In the presence of a strong magnetoelastic effect in SIO, any reorientations of magnetic moments under external fields can induce lattice distortions which, in turn, may result in sensible signals from electron transport measurements. ${ }^{11,21}$ Therefore, at low magnetic fields, where the uncompensated residual moments of $\mathrm{IrO}_{6}$ planes are not yet saturated (yielding no net moment), the observed 4-fold rotational symmetry AMR may be ascribed to the nature of the (non-distorted) tetragonal lattice structure, while the 2-fold AMR symmetry induced by large magnetic fields may be associated with field-induced metamagnetic transition of the magnetic order, thus demonstrating the emerging saturated state via the electron transport. Interestingly, as suggested by recent neutron scattering studies, ${ }^{23}$ there may exist twinning domains of orthorhombic magnetic structures in SIO, with magnetic moments in twinning domains having $90^{\circ}$ difference within the $\boldsymbol{a} \boldsymbol{b}$-plane. The PC AMR involves magnetic moments primarily from the local contact region $(\sim 1 \mu \mathrm{m})$, where the observations may be associated with a single-domain behavior. In such a scenario, the uniaxial magnetic anisotropy observed in PC measurements at higher fields may be induced when the canting of magnetic moments is tuned by an externally applied magnetic field. As for bulk AMR, multi-domain magnetic textures may be involved and possibly associated with the peculiar magnetic-field dependence. The abrupt shift of the AMR symmetry axis seen in the bulk measurement may be an indication of domain switching/realignments induced by the applied fields. The robust 2-fold AMR symmetry in the high field regime suggests that, under applied magnetic fields, one of the twinning magnetic domains is preferred over the other. The boundary field (of $\sim 150 \mathrm{mT}$ ) may correspond to the field needed for the reorientation.

In summary, point-contact investigations of magnetoelectronic transport in canted antiferromagnetic semiconductor $\mathrm{Sr}_{2} \mathrm{IrO}_{4}$ revealed large crystalline AMRs (up to 14\%) at liquid nitrogen temperature. Temperature dependent transport measurement found that $\mathrm{MR}$ vanishes at $\mathrm{T} \sim \mathrm{T}_{\text {Neel }}$, confirming the crucial role of magnetic order in magneto-electronic transport. Bulk and local (point contact) measurements of AMR exhibit qualitative differences, which may be attributed to the magnetic field effect on magnetic textures involved, including SIO twinning domains. The magnetic field dependence of AMR demonstrates strong correlations between electronic transport, magnetic properties and orbital states of this $5 d$ transition metal oxide. Our results suggest that the magnetic order in AFM spintronic devices can be probed by AMR as observed in $\mathrm{Sr}_{2} \mathrm{IrO}_{4}$.

This work was supported in part by C-SPIN, one of six centers of STARnet, a Semiconductor Research Corporation program, sponsored by MARCO and DARPA, and by NSF Grant Nos. DMR-1207577, DMR-1265162, and DMR1122603.

${ }^{1}$ W. H. Meiklejohn and C. P. Bean, Phys. Rev. 102, 1413 (1956).

${ }^{2}$ A. S. Núñez, R. A. Duine, P. Haney, and A. H. MacDonald, Phys. Rev. B 73, 214426 (2006). 
${ }^{3}$ A. H. MacDonald and M. Tsoi, Philos. Trans. R. Soc. London, Ser. A 369, 3098 (2011).

${ }^{4}$ J. Bass, A. Sharma, Z. Wei, and M. Tsoi, J. Magn. 13(1), 1 (2008).

${ }^{5}$ Z. Wei et al., Phys. Rev. Lett. 98, 116603 (2007).

${ }^{6}$ I. Fina et al., Nat. Commun. 5, 4671 (2014).

${ }^{7}$ B. G. Park et al., Nat. Mater. 10, 347-351 (2011).

${ }^{8}$ X. Marti et al., Phys. Rev. Lett. 108, 017201 (2012).

${ }^{9}$ B. J. Kim et al., Phys. Rev. Lett. 101, 076402 (2008).

${ }^{10}$ G. Cao et al., Phys. Rev. B 57, R11039(R) (1998).

${ }^{11}$ M. Ge et al., Phys. Rev. B 84, 100402(R) (2011).

${ }^{12}$ C. Wang et al., Phys. Rev. X 4, 041034 (2014).

${ }^{13}$ G. Cao et al., Phys. Rev. Lett. 78, 1751 (1997).
${ }^{14}$ Q. Huang et al., J. Solid State Chem. 112, 355 (1994).

${ }^{15}$ B. J. Kim et al., Science 323, 1329 (2009).

${ }^{16}$ A. G. M. Jansen, A. P. van Gelder, and P. Wyder, J. Phys. C 13, 6073 (1980).

${ }^{17}$ M. Tsoi et al., Phys. Rev. Lett. 80, 4281 (1998).

${ }^{18}$ A. A. Rushforth et al., Phys. Rev. Lett. 99, 147207 (2007).

${ }^{19}$ P. N. Hai et al., Appl. Phys. Lett. 100, 262409 (2012).

${ }^{20}$ Z. Ding et al., J. Appl. Phys. 113, 17B103 (2013).

${ }^{21}$ L. Miao, H. Xu, and Z. Q. Mao, Phys. Rev. B 89, 035109 (2014).

${ }^{22}$ F. Ye et al., Phys. Rev. B 87, 140406(R) (2013).

${ }^{23}$ C. Dhital et al., Phys. Rev. B 87, 144405 (2013).

${ }^{24}$ S. Boseggia et al., J. Phys.: Condens. Matter 25, 422202 (2013). 